\title{
ESTRADA INDEX OF GENERAL WEIGHTED GRAPHS
}

\author{
YILUN SHANG
}

(Received 12 June 2012; accepted 29 June 2012; first published online 28 September 2012)

\begin{abstract}
Let $G$ be a general weighted graph (with possible self-loops) on $n$ vertices and $\lambda_{1}, \lambda_{2}, \ldots, \lambda_{n}$ be its eigenvalues. The Estrada index of $G$ is a graph invariant defined as $E E=\sum_{i=1}^{n} e^{\lambda_{i}}$. We present a generic expression for $E E$ based on weights of short closed walks in $G$. We establish lower and upper bounds for $E E$ in terms of low-order spectral moments involving the weights of closed walks. A concrete example of calculation is provided.
\end{abstract}

2010 Mathematics subject classification: primary 05C50; secondary 05C22.

Keywords and phrases: Estrada index, weighted graph, spectral moment, graph spectrum.

\section{Introduction}

Throughout this paper we consider undirected graphs. Let $G$ be a simple graph (that is, unweighted and with no self-loops) on $n$ vertices. The eigenvalues of the adjacency matrix of $G$ form the spectrum of $G$. Let the spectrum of $G$ be $\lambda_{1}, \lambda_{2}, \ldots, \lambda_{n}$ arranged in nonincreasing order, that is,

$$
\lambda_{1} \geq \lambda_{2} \geq \cdots \geq \lambda_{n}
$$

The basic properties of graph spectrum can be found in the textbooks [1, 3]. A spectrum-based graph invariant introduced by Estrada [6-11] is defined by

$$
E E=E E(G)=\sum_{i=1}^{n} e^{\lambda_{i}},
$$

for which the name Estrada index is proposed [4].

The Estrada index has broad applicability in problems from the structure of longchain molecules and the degree of proteins [6-8, 11, 15] to the interdisciplinary field of complex (communication, social, metabolic) networks [9, 10, 17, 18, 20]. The literature contains a large collection of properties, especially upper and lower bounds, for the Estrada index (see, for example, [4, 5, 12-14, 16, 19, 21-23]).

(C) 2012 Australian Mathematical Publishing Association Inc. 0004-9727/2012 \$16.00 
However, most of them are tailored for simple graphs. Since real-life networks are closely related to weighted graphs which possibly contain some self-loops [2], it is of interest to study the Estrada index of general weighted graphs and its upper and lower bounds.

In this paper we address the Estrada index of general weighted graphs, where selfloops and even negative weights are allowed. Based on the spectral moments involving the weights of closed walks, we establish some upper and lower bounds for the Estrada index. In addition, we give a simple example to demonstrate the calculation of the Estrada index using our formulation.

The rest of the paper is organised as follows. In the next section we define graphtheoretical terminology and introduce the Estrada index for general weighted graphs. A useful lemma needed in our derivations is also presented. In Section 3 we use algebraic graph theory to derive upper and lower bounds on the Estrada index from the spectral moments. In Section 4 we use an example to demonstrate the calculation and to verify the bounds obtained in Section 3.

\section{Preliminaries}

Let $G=(\mathcal{V}, \mathcal{E}, \mathcal{W})$ be a weighted graph, where $\mathcal{V}=\left\{v_{1}, \ldots, v_{n}\right\}$ denotes a set of $n$ nodes, $\mathcal{E} \subseteq \mathcal{V} \times \mathcal{V}$ denotes a set of undirected edges, and $\mathcal{W}=\left\{w_{i j} \in \mathbb{R}\right.$ : for all $\left.\left\{v_{i}, v_{j}\right\} \in \mathcal{E}\right\}$ is the set of (possibly negative) weights. The weighted adjacency matrix of $G$, defined by $W=\left(w_{i j}\right)$, is an $n \times n$ symmetric matrix where $w_{i j}=0$ if $v_{i}$ is not adjacent to $v_{j}$. We order the eigenvalues of $W$ as $\lambda_{1} \geq \lambda_{2} \geq \cdots \geq \lambda_{n}$ [3].

For $k=0,1,2, \ldots$, let $M_{k}=M_{k}(G)$ be the $k$ th spectral moment of a weighted graph $G$,

$$
M_{k}=\sum_{i=1}^{n} \lambda_{i}^{k}
$$

The Estrada index of $G$ is also defined as in (1.1). Bearing in mind the power expansion of $e^{x}$, we obtain

$$
E E(G)=\sum_{k \geq 0} \frac{M_{k}(G)}{k !}
$$

A walk of length $k$ from $v_{i_{1}}$ to $v_{i_{k+1}}$ is an ordered sequence of nodes $\left(v_{i_{1}}, v_{i_{2}}, \ldots, v_{i_{k+1}}\right)$ such that $\left\{v_{i_{j}}, v_{i_{j+1}}\right\} \in \mathcal{E}$ for $j=1,2, \ldots, n$. The walk is said to be closed if $v_{i_{1}}=v_{i_{k+1}}$. Given a walk $s=\left(v_{i_{1}}, v_{i_{2}}, \ldots, v_{i_{k+1}}\right)$ in a weighted graph $G$, we define the weight of the walk as $w(s)=w_{i_{1} i_{2}} w_{i_{2} i_{3}} \cdots w_{i_{k} i_{k+1}}$. Denote by $K_{n}$ the complete graph on $n$ vertices, and by $J_{n}$ the corresponding complete loopy graph, that is, $K_{n}$ with a self-loop on each node. Recall that, for a simple graph $G, M_{k}(G)$ is the number of closed walks of length $k$ in the graph [3]. In the case of weighted graphs, we generalise the result in terms of weights of closed walks as follows. 
LEMMA 2.1. Let $G=(\mathcal{V}, \mathcal{E}, \mathcal{W})$ be a weighted graph with weighted adjacency matrix $W=\left(w_{i j}\right)$. Then

$$
M_{k}=\sum_{i=1}^{n} \sum_{s \in S_{k}^{(i)}} w(s),
$$

where $S_{k}^{(i)}$ is the set of closed walks of length $k$ from $v_{i}$ to itself in the complete loopy graph $J_{n}$.

Proof. By direct calculation,

$$
\begin{aligned}
\left(W^{k}\right)_{i i} & =\sum_{i=1}^{n} \sum_{i_{2}=1}^{n} \cdots \sum_{i_{k}=1}^{n} w_{i i_{2}} w_{i_{2} i_{3}} \cdots w_{i_{k} i} \\
& =\sum_{s \in S_{k}^{(i)}} w(s) .
\end{aligned}
$$

Since $W$ is symmetric and diagonalisable,

$$
M_{k}=\sum_{i=1}^{n} \lambda_{i}^{k}=\operatorname{Trace}\left(W^{k}\right)=\sum_{i=1}^{n} \sum_{s \in S_{k}^{(i)}} w(s),
$$

as desired.

It follows from (2.1) and (2.2) that the Estrada index of a weighted graph $G$ can be expressed as

$$
E E=\sum_{k \geq 0} \sum_{i=1}^{n} \sum_{s \in S_{k}^{(i)}} \frac{w(s)}{k !} .
$$

As is known, the Estrada index of a simple graph $G$ changes monotonically when edges are added or deleted [4]. In particular,

$$
E E\left(\overline{K_{n}}\right) \leq E E(G) \leq E E\left(K_{n}\right),
$$

where $\overline{K_{n}}$ is the complement of $K_{n}$. However, from (2.3) it is easy to see that the Estrada index of a weighted graph is no longer monotonic and (2.4) breaks down since we may have negative edge weights. Generally, when $G$ is a weighted graph, as we shall show in Section 3, the bounds for $E E(G)$ are much more complicated than those found for simple graphs.

\section{Bounds for the Estrada index}

In this section we present our upper and lower bounds for the Estrada index of $G$ using the weights of closed walks. Clearly, if $G$ is a null graph (that is, an edgeless graph $\left.\overline{K_{n}}\right)$, then $E E(G)=n$. In the sequel, we assume that $G$ contains at least one edge. 
Theorem 3.1. Let $G=(\mathcal{V}, \mathcal{E}, \mathcal{W})$ be a weighted graph with weighted adjacency matrix $W=\left(w_{i j}\right)$. Let $\varepsilon=\min \left\{\left|w_{i j}\right|: 1 \leq i \leq j \leq n, w_{i j} \neq 0\right\}$. Assume that $G$ contains at least one edge. Then

$$
\begin{aligned}
& \sum_{k=0}^{3} \sum_{i=1}^{n} \sum_{s \in S_{k}^{(i)}} \frac{w(s)}{k !}+\frac{1}{\varepsilon^{4}}\left(\cosh (\varepsilon)-1-\frac{\varepsilon^{2}}{2 !}\right) \sum_{i=1}^{n} \sum_{s \in S_{4}^{(i)}} w(s) \\
& +\frac{1}{\varepsilon^{5}}\left(\sinh (\varepsilon)-\varepsilon-\frac{\varepsilon^{3}}{3 !}\right) \sum_{i=1}^{n} \sum_{s \in S_{5}^{(i)}} w(s) \leq E E(G) \\
& \leq n-1+\exp \left(\sqrt{\left.-1+\sum_{i=1}^{n} \sum_{s \in S_{2}^{(i)}} w(s)\right) .}\right.
\end{aligned}
$$

Proof. We first consider the lower bound. We claim that, for all $k \geq 4$,

$$
M_{k+2} \geq \varepsilon^{2} M_{k}
$$

To see this, we note that by repeating the first edge twice for a closed walk of length $k$, we get a closed walk of length $k+2$. Thus (3.2) follows easily from Lemma 2.1.

By virtue of (2.1), (2.3) and (3.2),

$$
\begin{aligned}
E E(G)= & \sum_{k=0}^{3} \sum_{i=1}^{n} \sum_{s \in S_{k}^{(i)}} \frac{w(s)}{k !}+\sum_{k \geq 2} \frac{M_{2 k}}{(2 k) !}+\sum_{k \geq 2} \frac{M_{2 k+1}}{(2 k+1) !} \\
\geq & \sum_{k=0}^{3} \sum_{i=1}^{n} \sum_{s \in S_{k}^{(i)}} \frac{w(s)}{k !}+\sum_{k \geq 2} \frac{\left(\varepsilon^{2}\right)^{k-2} M_{4}}{(2 k) !}+\sum_{k \geq 2} \frac{\left(\varepsilon^{2}\right)^{k-2} M_{5}}{(2 k+1) !} \\
\geq & \sum_{k=0}^{3} \sum_{i=1}^{n} \sum_{s \in S_{k}^{(i)}} \frac{w(s)}{k !}+\sum_{k \geq 2} \frac{\varepsilon^{2 k} M_{4}}{\varepsilon^{4}(2 k) !}+\sum_{k \geq 2} \frac{\varepsilon^{2 k+1} M_{5}}{\varepsilon^{5}(2 k+1) !} \\
= & \sum_{k=0}^{3} \sum_{i=1}^{n} \sum_{s \in S_{k}^{(i)}} \frac{w(s)}{k !}+\frac{M_{4}}{\varepsilon^{4}}\left(\cosh (\varepsilon)-1-\frac{\varepsilon^{2}}{2 !}\right) \\
& +\frac{M_{5}}{\varepsilon^{5}}\left(\sinh (\varepsilon)-\varepsilon-\frac{\varepsilon^{3}}{3 !}\right) .
\end{aligned}
$$

Combining this with (2.2), we obtain the lower bound. 
Next, we turn to the proof of the upper bound. Since the graph $G$ contains at least one edge, $G$ has at least one positive and at least one negative eigenvalue [3]. We denote by $n_{+}$the number of positive eigenvalues of $G$.

In view of the monotonicity of $f(x)=e^{x}$ in the interval $(-\infty,+\infty)$, we obtain

$$
\begin{aligned}
E E(G) & =\sum_{i=1}^{n} e^{\lambda_{i}} \leq n-n_{+}+\sum_{i=1}^{n_{+}} e^{\lambda_{i}} \\
& =n-n_{+}+\sum_{i=1}^{n_{+}} \sum_{k \geq 0} \frac{\lambda_{i}^{k}}{k !} \\
& =n+\sum_{k \geq 1} \frac{1}{k !} \sum_{i=1}^{n_{+}}\left(\lambda_{i}^{2}\right)^{k / 2} \\
& \leq n+\sum_{k \geq 1} \frac{1}{k !}\left(\sum_{i=1}^{n_{+}} \lambda_{i}^{2}\right)^{k / 2} \\
& =n+\sum_{k \geq 1} \frac{1}{k !}\left(M_{2}-\sum_{i=n_{+}+1}^{n} \lambda_{i}^{2}\right)^{k / 2} \\
& =n+\sum_{k \geq 1} \frac{1}{k !}\left(\sum_{i=1}^{n} \sum_{s \in S_{2}^{(i)}} w(s)-\sum_{i=n_{+}+1}^{n} \lambda_{i}^{2}\right)^{k / 2}
\end{aligned}
$$

where in the last equality we use Lemma 2.1.

Note that $G$ must have $K_{2}$ as an induced subgraph, whose spectrum is 1 and -1 . It follows from the interlacing theorem [1] that $\lambda_{n} \leq-1$, which indicates that $\sum_{i=n_{+}+1}^{n} \lambda_{i}^{2} \geq 1$. Therefore, from (3.3),

$$
\begin{aligned}
E E(G) & \leq n+\sum_{k \geq 1} \frac{1}{k !}\left(-1+\sum_{i=1}^{n} \sum_{s \in S_{2}^{(i)}} w(s)\right)^{k / 2} \\
& =n-1+\exp \left(\sqrt{-1+\sum_{i=1}^{n} \sum_{s \in S_{2}^{(i)}} w(s)}\right)
\end{aligned}
$$

as desired.

We remark here that if $G$ is a simple graph, then the weighted adjacency matrix $W$ reduces to the ordinary $0-1$ adjacency matrix $A$. Then $\varepsilon=1$, and the above lower bound of $E E$ matches [22, Proposition 2]. Furthermore, it is easy to see that the above upper bound is also consistent with [16, Theorem 2.1], which is better than the upper bound in [4, Theorem 1]. 


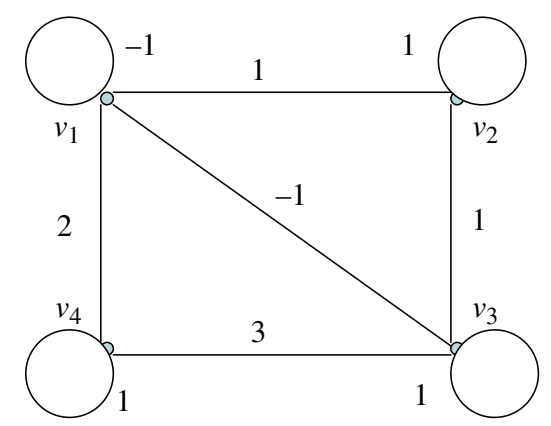

Figure 1. A weighted graph $G$ on four nodes with $w_{11}=w_{13}=-1, w_{12}=w_{22}=w_{23}=w_{33}=w_{44}=1$, $w_{14}=2$ and $w_{34}=3$.

\section{An example}

In this section, we present a concrete example. In Figure 1 we have a weighted graph $G=(\mathcal{V}, \mathcal{E}, \mathcal{W})$ with four nodes, whose weighted adjacency matrix is

$$
W=\left(\begin{array}{rrrr}
-1 & 1 & -1 & 2 \\
1 & 1 & 1 & 0 \\
-1 & 1 & 1 & 3 \\
2 & 0 & 3 & 1
\end{array}\right)
$$

The eigenvalues of $W$ are given by $\lambda_{1}=4.33, \lambda_{2}=1.13, \lambda_{3}=0.52$ and $\lambda_{4}=-3.97$.

It follows from the definition (1.1) that $E E(G)=\sum_{i=1}^{4} e^{\lambda_{i}}=80.41$. To obtain the upper and lower bounds given in Theorem 3.1, we first need to calculate the first several spectral moments. By using Lemma 2.1, we obtain $M_{0}=4$,

$$
\begin{aligned}
& M_{1}=\sum_{i=1}^{4} \sum_{s \in S_{1}^{(i)}} w(s)=-1+1+1+1=2 \\
& M_{2}=\sum_{i=1}^{4} \sum_{s \in S_{2}^{(i)}} w(s)=7+3+12+14=36 \\
& M_{3}=\sum_{i=1}^{4} \sum_{s \in S_{3}^{(i)}} w(s)=-16+0+6+6=-4,
\end{aligned}
$$

and similarly, $M_{4}=42$ and $M_{5}=18$. By definition, we have $\varepsilon=1$. Hence, from (3.1) we derive the upper and lower bounds as 373.95 and 39.96 , respectively. It seems that the lower bound is relatively tighter than the upper bound for this specific example.

\section{Open problems}

We conclude the paper by raising some open problems. Can we characterise the effect of weights on $E E$ in a more succinct way (presumably less accurate)? If we 
define some cost functions for the difference between the upper and lower bounds, how can we determine the optimal values of weights?

\section{Acknowledgements}

The author is grateful to the editor and the anonymous referee for valuable suggestions and comments.

\section{References}

[1] N. Biggs, Algebraic Graph Theory (Cambridge University Press, Cambridge, 1993).

[2] G. Caldarelli and A. Vespignani, Large Scale Structure and Dynamics of Complex Networks: From Information Technology to Finance and Natural Science (World Scientific, Singapore, 2007).

[3] D. M. Cvetković, M. Doob, I. Gutman and A. Torgašev, Recent Results in the Theory of Graph Spectra (North-Holland, Amsterdam, 1988).

[4] J. A. de la Peña, I. Gutman and J. Rada, 'Estimating the Estrada index', Linear Algebra Appl. 427 (2007), 70-76.

[5] Z. Du and B. Zhou, 'The Estrada index of trees', Linear Algebra Appl. 435 (2011), 2462-2467.

[6] E. Estrada, 'Characterization of 3D molecular structure', Chem. Phys. Lett. 319 (2000), 713-718.

[7] E. Estrada, 'Characterization of the folding degree of proteins', Bioinformatics 18 (2002), 697-704.

[8] E. Estrada, 'Characterization of the amino acid contribution to the folding degree of proteins', Proteins 54 (2004), 727-737.

[9] E. Estrada and J. A. Rodríguez-Velázquez, 'Subgraph centrality in complex networks', Phys. Rev. E 71 (2005), 056103.

[10] E. Estrada and J. A. Rodríguez-Velázquez, 'Spectral measures of bipartivity in complex networks', Phys. Rev. E 72 (2005), 046105.

[11] E. Estrada, J. A. Rodríguez-Velázquez and M. Randić, 'Atomic branching in molecules', Int. J. Quantum Chem. 106 (2006), 823-832.

[12] I. Gutman, 'Lower bounds for Estrada index', Publ. Inst. Math. Beograd (N.S.) 83 (2008), 1-7.

[13] I. Gutman and A. Graovac, 'Estrada index of cycles and paths', Chem. Phys. Lett. 436 (2007), 294-296.

[14] I. Gutman and S. Radenković, 'A lower bound for the Estrada index of bipartite molecular graphs', Kragujevac J. Sci. 29 (2007), 67-72.

[15] A. Ilić and D. Stevanović, 'The Estrada index of chemical trees', J. Math. Chem. 47 (2010), 305-314.

[16] J. Liu and B. Liu, 'Bounds of the Estrada index of graphs', Appl. Math. J. Chinese Univ. 25 (2010), 325-330.

[17] Y. Shang, 'Perturbation results for the Estrada index in weighted networks', J. Phys. A: Math. Theor. 44 (2011), 075003.

[18] Y. Shang, 'Local natural connectivity in complex networks', Chin. Phys. Lett. 28 (2011), 068903.

[19] Y. Shang, 'The Estrada index of random graphs', Sci. Magna 7 (2011), 79-81.

[20] Y. Shang, 'Biased edge failure in scale-free networks based on natural connectivity', Indian J. Phys. 86 (2012), 485-488.

[21] B. Zhou, 'On Estrada index', MATCH Commun. Math. Comput. Chem. 60 (2008), 485-492.

[22] B. Zhou and Z. Du, 'Some lower bounds for Estrada index', Iran. J. Math. Chem. 1 (2010), 67-72.

[23] B. Zhou and N. Trinajstić, 'Estrada index of bipartite graphs', Int. J. Chem. Model 1 (2008), 387-394.

YILUN SHANG, Institute for Cyber Security, University of Texas at San Antonio, San Antonio, Texas 78249, USA e-mail: shylmath@hotmail.com 